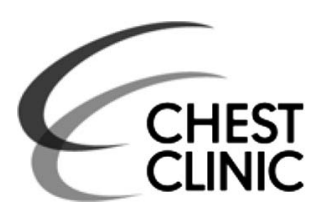

CASE BASED DISCUSSION

\title{
It ain't necessarily so: a surprising lower airway infection
}

\author{
Rossa Brugha, ${ }^{1,2}$ Anne Hall, ${ }^{3}$ Andrew Bush ${ }^{1,2}$
}

${ }^{1}$ National Heart and Lung Institute, Imperial College London, London, UK ${ }^{2}$ Department of Paediatric Respiratory Medicine, Royal Brompton Hospital, London, UK

${ }^{3}$ Department of Microbiology, Royal Brompton Hospital, London, UK

\section{Correspondence to} Dr Rossa Brugha, Population Health and Gene Therapy, National Heart and Lung Institute, Imperial College London, Emmanuel Kaye Building, Manresa Road, London SW3 6LR, UK; r.brugha@imperial.ac.uk

Received 24 July 2016 Revised 14 September 2016 Accepted 4 October 2016 Published Online First 31 October 2016
Rossa Brugha (Junior Doctor): A 3-year-old boy with cystic fibrosis (CF) diagnosed on newborn screening (sweat chloride $90 \mathrm{mmol} / \mathrm{L}$, F508del homozygous) attended routine follow-up in the CF clinic. He had had a wet cough for the previous 3 weeks. In addition to CF, he had previously been diagnosed with gastro-oesophageal reflux (oesophageal $\mathrm{pH}<4.0$ for $13 \%$ of study at age 2 months) and an unsafe swallow with risk of aspiration on thin fluids (on videofluoroscopy at age 23 months). $\mathrm{He}$ had completed a 2-week course of co-amoxiclav $400 / 575 \mathrm{~mL}$ twice daily and then started a 2-week course of ciprofloxacin $250 \mathrm{mg}$ twice daily in view of a previous growth of Pseudomonas aeruginosa at 14 months of age. He was not wheezing but his mother said that she could feel crackling in his chest. He was otherwise well, with no fever or coryzal symptoms. A cough swab taken at this clinic showed no bacterial growth. Three months prior to this he had similar symptoms, during which an induced sputum grew Haemophilus influenzae, which was ampicillin resistant and treated with a course of cefixime.

Andrew Bush (Professor of Respiratory Paediatrics): In summary, this preschool child with $\mathrm{CF}$ has ongoing symptoms despite appropriate empiric courses of antibiotics. At this point, the differential includes CF-related issues and coincident disease. In a child with $\mathrm{CF}$, ongoing undiagnosed and untreated infection is the likeliest diagnosis, but other causes should be considered, including undiagnosed allergic bronchopulmonary aspergillosis (ABPA), persistently symptomatic gastro-oesophageal reflux despite medical therapy (which is more common in CF than in normal children) and upper airway disease leading to postnasal drip. CF-related diabetes should always be considered even in young children as a comorbidity if the clinical course is not straightforward. In this particular child, issues unrelated to CF particularly include ongoing aspiration on syrup consistency fluids, but other causes of chronic cough, ${ }^{1}$ for example, TB and endobronchial foreign body must not be forgotten. It should also be remembered that a simple cold in a normal child may cause symptoms lasting more than 3 weeks. ${ }^{2}$

RB: Clinical examination excluded common childhood causes for persistent cough such as tonsillitis or viral-induced wheeze. Proton pump inhibitor therapy was already maximal for his age, and he was not given an additional $\mathrm{H}_{2}$ blocker. ABPA markers were normal, with negative aspergillus $\mathrm{IgG}$, Aspergillus fumigatus RAST $0.1 \mathrm{IU} / \mathrm{mL}$ and a total IgE of $25 \mathrm{IU} / \mathrm{mL}$. Random blood glucose readings were 4.2 and $4.6 \mathrm{mmol} / \mathrm{L}(75-83 \mathrm{mg} / \mathrm{dL})$. In view of possible ongoing aspiration, he was referred again to the speech and language therapy team for a repeat assessment. He was reviewed after 4 weeks in outpatients by a consultant who again noted a wet cough, which occasionally had an added wheezing note. However, at this point, he had a runny nose. Another course of co-amoxiclav was given, with inhaled salbutamol for wheezing symptoms, and a videofluoroscopy arranged. This again showed a risk of aspiration with thin fluids, with a safe effective swallow on syrup-thick fluids. Cough swab at clinic again was negative.

AB: After 4 weeks I reviewed him in clinic. $\mathrm{He}$ looked sleepy and 'not himself', with an ongoing cough, a hoarse voice and a respiratory rate of 48 breaths per minute while asleep. Chest radiograph (figure 1) showed bronchial wall thickening in the right lower zone and behind the heart. By this time he had been coughing for nearly 2 months, with no diagnosis. He was admitted for bronchoscopy and intravenous antibiotics.

RB: Bronchoscopy revealed anatomically normal airways but with friable inflamed mucosa and creamy yellow secretions. Cell differential demonstrated a neutrophilia (64\%). Microbiological samples identified $H$. influenzae on bacterial culture, and a PCR assay of the lavage fluid, for nucleic acids from common respiratory viruses, was positive for influenza A RNA. He was treated with intravenous ceftazidime and tobramycin, oral oseltamivir and isolated from other inpatients. On subsequent discussion with his mother, we learnt he had received the nasal influenza vaccination (live attenuated influenza vaccine (LAIV)) 2 days prior to the bronchoscopy.

Anne Hall (Consultant Microbiologist): Sequence analysis at the reference laboratory demonstrated that the virus isolated showed 99\%-100\% genetic homology to the influenza $\mathrm{H} 3$ strain included in the 2015/2016 LAIV. The LAIV has greater efficacy than the inactivated vaccine in children aged 16 years and is safe in $\mathrm{CF}^{3}$ Shedding of LAIV from nasal samples is reported in $44 \%$ of children aged 5-9 years for a mean of 2 days postadministration ${ }^{4}$ and this proportion decreases in older children and adults. ${ }^{4}$ To our knowledge, this is the first report of a positive bronchoalveolar lavage sample in a child with CF who recently received the LAIV. It is a true-positive microbiological test in the context of what is essentially a clinically false-positive result; we initially attributed his cough to a prolonged viral chest exacerbation with influenza.

RB: We report this case to avoid others making the same mistake. The lesson we learnt is to take a 


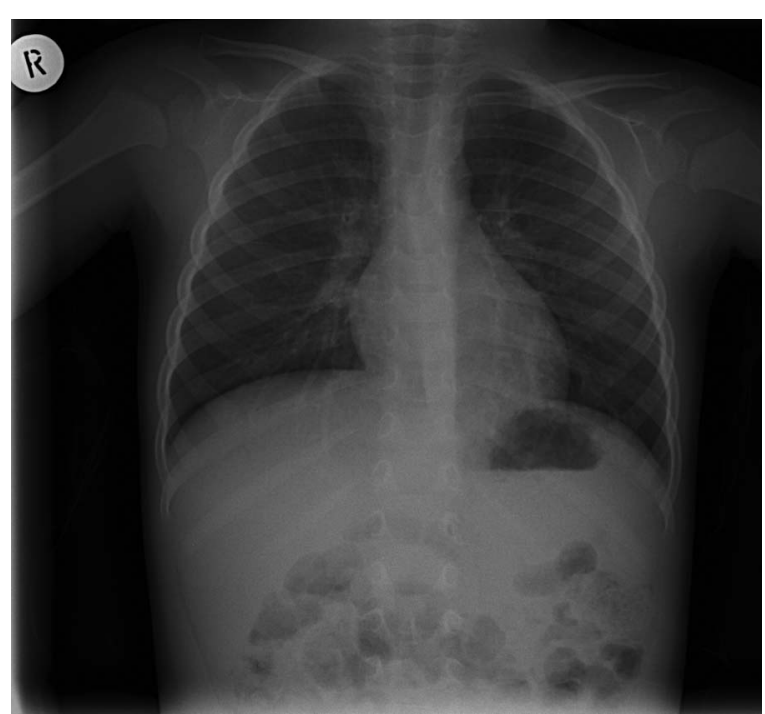

Figure 1 Chest radiograph demonstrating bilateral bronchial wall thickening.

comprehensive vaccine history when taking microbiological samples and that in this era of live inhaled vaccines, a live attenuated vaccine may cause a false-positive result. It is possible that we did harm to this child with an unnecessary medication by giving a neuraminidase inhibitor in the context of a recent immunisation. In addition, he was isolated from other non-CF patients unjustifiably. The US Centre for Disease Control guidelines advise that LAIV should not be administered until
48 hours after the cessation of antiviral drugs active against influenza, ${ }^{5}$ and it is possible that we have attenuated our patient's immune response to the influenza vaccination. His wet cough has improved on thickened fluids and we have attributed the cough to his unsafe swallow. Investigations into the aetiology of this are ongoing.

AB: So in the words of Ira Gershwin in Porgy and Bess, in this case definitely 'It ain't necessarily so'.

Twitter Follow Rossa Brugha at @_rossa_

Contributors $\mathrm{RB}$ conceived the report and wrote the first draft with $\mathrm{AB}$. $\mathrm{AH}$ provided expert commentary. All authors approved the submission.

Funding $A B$ was supported by the NIHR Respiratory Disease Biomedical Research Unit at the Royal Brompton and Harefield NHS Foundation Trust and Imperial College London. RB is supported by an NIHR Clinical Lectureship.

Competing interests None.

Patient consent Obtained.

Provenance and peer review Not commissioned; externally peer reviewed.

\section{REFERENCES}

1 Shields MD, Bush A, Everard ML, et al. Recommendations for the assessment and management of cough in children. Thorax 2008;63:iii1-15.

2 Stokholm J, Chawes BL, Vissing NH, et al. Azithromycin for episodes with asthma-like symptoms in young children aged 1-3 years: a randomised, double-blind placebo-controlled trial. Lancet Respir Med 2016;4:19-26.

3 Boikos C, De Serres G, Lands LC, et al. Safety of live-attenuated influenza vaccination in cystic fibrosis. Pediatrics 2014;134:e983-91.

4 Block SL, Yogev R, Hayden FG, et al. Shedding and immunogenicity of live attenuated influenza vaccine virus in subjects $5-49$ years of age. Vaccine 2008;26:4940-6.

5 https://www.cdc.gov/vaccines/pubs/pinkbook/downloads/genrec.pdf 